well documented. AHAVACC guidelines recommend aspirin in all cases of ACS; however, it has been suggested that the bleeding risks associated with aspirin might outweigh the benefits of this therapy in patients with thrombocytopenia. Sarkiss et al. have evaluated aspirin therapy in cancer patients with ACS and thrombocytopenia secondary to chemotherapy or bone marrow suppression.

The authors retrospectively reviewed the records of 70 patients with cancer who had been diagnosed with ACS and referred for cardiology consultation. The 27 patients with thrombocytopenia (platelet counts $\leq 1.0 \times 10^{11} / \mathrm{l}$ ) had worse 7 -day survival than the 43 patients without thrombocytopenia (37\% vs $77 \%$; $P=0.0012)$. Among the nonthrombocytopenic patients, aspirin therapy was associated with improved 7-day survival compared with no aspirin therapy (88\% vs $45 \% ; P=0.0096)$. Notably, aspirin therapy was also associated with better 7-day survival in those patients with thrombocytopenia ( $90 \%$ vs $6 \%$; $P<0.0001)$. No major adverse bleeding events were observed; minor bleeding was seen in 12 patients, but was not associated with aspirin use.

Aspirin could, therefore, be beneficial in cancer patients with ACS with or without thrombocytopenia. The authors also report similar observed benefits of $\beta$-blocker use on 7-day survival in patients with and without thrombocytopenia. They do warn, however, that clinicians should consider individual cases carefully, as the potential for serious bleeding complications in thrombocytopenia remains high.

Original article Sarkiss MG et al. (2007) Impact of aspirin therapy in cancer patients with thrombocytopenia and acute coronary syndromes. Cancer 109: 621-627

\section{Factors underlying superior hypertension control in the US}

Guidelines for the treatment of hypertension are more aggressive in the US than elsewhere. To determine whether differences in guidelines translate into differences in clinical variables, Wang et al. analyzed data for the year 2004 from CardioMonitor-an ongoing, nationally representative survey of physician visits made by patients with cardiovascular diseases. The data set included 21,053 adults with hypertension who had visited 1,284 primary care physicians and 291 cardiologists in France, Germany, Italy, Spain, the UK or the US.

A minimum of $92 \%$ of patients received drug treatment for hypertension in each country. Average pretreatment blood pressure was lower in the US than in the European countries (161/94 mmHg vs 167-173/96-99 mmHg), while concomitant use of antihypertensive drugs from two or more classes was more common in the US (64\% vs $44-59 \%$ of patients).

The authors defined 'controlled hypertension' as a most-recent blood pressure reading of less than $140 / 90 \mathrm{mmHg}$. Multivariate analyses showed that hypertension was less likely to be controlled in the European countries (odds ratios 0.27-0.50; $P<0.001$ for all). The likelihood of a dose escalation or medication change in response to inadequately controlled hypertension was also lower in Europe (odds ratios $0.29-0.65 ; P<0.001$ for all).

The better rates of hypertension control in the US seem, therefore, to be attributable to lower thresholds for initiating treatment, and more-intensive drug regimens.

Original article Wang YR et al. (2007) Outpatient

hypertension treatment, treatment intensification, and control in Western Europe and the United States. Arch Intern Med 167: $141-147$

\section{Symptomatic venous thromboembolism risk decreases during anticoagulant prophylaxis}

Anticoagulant prophylaxis is highly recommended in medical patients who are at risk of venous thromboembolism (including pulmonary embolism and deep venous thrombosis), but is underutilized. Dentali et al. hypothesized that this reluctance could be caused by a lack of evidence that prophylaxis prevents clinically important outcomes in these patients. To examine whether anticoagulant prophylaxis reduces venous thromboembolism in at-risk patients, they performed a meta-analysis of available randomized clinical trials.

The authors searched the MEDLINE, EMBASE and Cochrane databases to September 2006 for randomized trials comparing anticoagulant prophylaxis with no treatment in hospitalized medical patients. Nine studies (19,958 patients) were identified, seven of which were double-blind. Pulmonary embolism and fatal pulmonary embolism occurrence 